\title{
Betriebliche Beschäftigungssicherung durch interne Personalvermittlung
}

In der jüngsten Wirtschafts- und Finanzkrise wurde eindrucksvoll demonstriert, wie flexibel interne Arbeitsmärkte auf krisenhafte Situationen reagieren können, wenn geeignete institutionelle Rahmenbedingungen gegeben sind. Die Diskussion konzentrierte sich auf die Instrumente konjunkturelle Kurzarbeit, die Pufferwirkung von Arbeitszeitkonten und, schon eher am Rande, Modelle zur zeitweisen tariflichen Absenkung der Arbeitszeit. Wenig beachtet wurde und wird die interne Flexibilität, die durch die Personalvermittlung auf internen Arbeitsmärkten bereitgestellt wird. Auf dieses betriebliche Flexibilitätsinstrument - auf Abteilungen zur internen Personalvermittlung - konzentriert sich der folgende Beitrag.

\section{Einleitung}

Die Debatte um die Bewältigung von Unternehmenskrisen hat im September 2010 einen aktuellen Impuls erhalten. Mit der Standort- und Beschäftigungssicherung des Siemens-Konzerns ist das erste Mal seit Langem das Thema Restrukturierung im Zusammenhang mit Beschäftigungssicherung im Unternehmen und damit positiv thematisiert worden. Der Technologiekonzern will zukünftig - neben Kurzarbeit interne Personalbewegungen zwischen seinen Standorten organisieren, um das vereinbarte Ziel einhalten zu können, auch in Restrukturierungsfällen keine betriebsbedingten Kündigungen auszusprechen. Diese Initiative ist gut und erfreulich, und sie bildet einen Kontrast zum üblichen Diskurs über Restrukturierungen mit dem Fokus auf Massenentlassungen als Managementfehler oder Betriebsunfall. Das Versprechen des Unternehmens, auf unbestimmte Zeit auf betriebsbedingte Kündigungen zu verzichten und damit andere Wege zu gehen als den der konventionellen Restrukturierung-Entlassung plus Abfindung-, wird vor diesem Hintergrund als innovativ gekennzeichnet.

Siemens gilt als Innovator, weil in der Vergangenheit die innerbetriebliche Beschäftigungssicherung für die arbeitsmarktpolitische Öffentlichkeit weitgehend unsichtbar gewirkt hat. Tatsächlich sind die Instrumente weiter erprobt und verbreitet als landläufig angenommen wird. Eine Schlüsselstelle nimmt dabei die innerbetriebliche Arbeits- und Personalvermittlung ein. Viele Unternehmen haben eigene, interne Organisationen aufgebaut mit dem
Ziel, den Abbau von Stellen zu bewältigen, ohne die betroffenen Beschäftigten auf den externen Arbeitsmarkt zu entlassen und den damit verbundenen sozialen Risiken auszusetzen. Für diese Organisationen möchten wir den Begriff „Abteilungen zur internen Personalvermittlung " einführen (vgl. Kirsch/Mühge 2010). Die Abteilungen selbst bezeichnen sich häufig als „internes Arbeitsamt unseres Unternehmens", da sich ihre Aufgabe gut mit der der örtlichen Arbeitsagentur vergleichen lässt: Personalvermittlungsabteilungen beraten, qualifizieren und vermitteln Beschäftigte, die ihren Arbeitsplatz verloren haben, auf offene Stellen des internen Arbeitsmarkts.

Der Beitrag gliedert sich im Folgenden in drei Teile. Abschnitt 2 geht der Hypothese nach, dass die interne Personalvermittlung einen signifikanten Beitrag zur Flexibilität von Unternehmen und zur Beschäftigungssicherung auf internen Arbeitsmärkten leistet. Sind Personalvermittlungsabteilungen ein effektives Instrument zur Bewältigung des Strukturwandels auf internen Arbeitsmärkten, oder sind sie lediglich symbolische Personalpolitik? Der Beitrag geht von der These aus, dass Personalvermittlungsabteilungen ein hohes Niveau an Beschäftigungssicherung leisten, woran zwei weitere Fragen angeschlossen werden: Auf welche Weise stehen Regeln zum betrieblichen Kündigungsschutz im Zusammenhang mit Personalvermittlungsabteilungen? Und eng damit verbunden - inwiefern entstehen jenseits der beschäftigungssichernden Wirkung von Personalvermittlungsabteilungen neue Risiken für die Beschäftigten?

In Abschnitt 3 konzentrieren wir uns auf die organisatorischen innerbetriebli- chen Voraussetzungen und Probleme der internen Arbeitsvermittlung. Wir gehen von einer Interessendivergenz bei verschiedenen Akteuren des internen Arbeitsmarkts aus, die durch die interne Personalvermittlung ausgelöst wird. Personalversetzungsabteilungen sind darauf angewiesen, diese Interessendivergenz zu überwinden. Hierzu benötigen sie hinreichende Geld-, Macht- und Personalressourcen.

Abschnitt 4 stellt schließlich dar, dass die Arbeit von Personalversetzungsabteilungen verschiedene Formen der Beschäftigungslosigkeit auf internen Arbeitsmärkten aufdeckt. Empirische Grundlage der Ausführungen sind quantitative und qualitative Daten aus zwei Forschungsprojekten. ${ }^{1}$ Abschnitt 5 schließt den Beitrag mit einem positiven Fazit der Arbeit von Personalvermittlungsabteilungen ab.

\footnotetext{
Die quantitativen Daten stammen aus dem abgeschlossenen Forschungsprojekt „WEGA - Wirksamkeit von Einrichtungen zur Gestaltung interner Arbeitsmärkte", gefördert durch die Mitbestimmungsförderung der Hans-Böckler-Stiftung; die qualitativen Ergebnisse stammen ferner aus dem laufenden Forschungsvorhaben „BOPS - Beruflichkeit, Organisations- und Personalentwicklung im Spannungsfeld von Restrukturierung und Kompetenzsicherung “, gefördert von BMBF/DLR, Projektträger Arbeitsgestaltung und Dienstleistung, Förderkennzeichen 01FH09016.
}

Gernot Mühge, Dipl.-Sozialwissenschaftler, forscht am Institut Arbeit und Qualifikation (IAQ) der Universität Duisburg-Essen. Arbeitsschwerpunkte: Unternehmensrestrukturierung, Interne Arbeitsmärkte und Beschäftigtentransfer. e-mail: gernot.muehge@uni-due.de 
Verbreitung und Wirksamkeit der internen Arbeitsvermittlung

Die Verbreitung und Wirksamkeit von Personalvermittlungsabteilungen (PVA) wurden im Jahr 2008 durch zwei Befragungen untersucht. Die erste Befragung hatte Führungskräfte aus PVA als Zielpersonen; inhaltlich standen Struktur und Effektivität ihrer Abteilungen im Zentrum. Eine zweite Befragung zielte auf Betriebs- und Personalräte, in deren Zuständigkeit die PVA fällt; mit diesem Fragebogen wurden vor allem der Verhandlungsprozess um die PVA und mögliche Risiken für die Beschäftigten thematisiert. ${ }^{2}$

\subsection{VERBREITUNG}

Im Vorfeld der Befragungen stand eine mehrmonatige Recherchephase, da zu Beginn der Forschungsarbeiten im Jahr 2006 nur wenige Unternehmen mit eigenen Abteilungen zur internen Personalvermittlung bekannt waren. Hinweise aus der Forschung kamen aus frühen Arbeiten zu Beschäftigungsplänen und betrieblichen Strategien zu „Qualifizieren statt Entlassen“ (Bosch 1990); weiterhin lagen Fallstudien zu einzelnen Unternehmen vor. In erster Linie war (und ist) das Bild von Personalvermittlungsabteilungen durch wenige Einzelbeispiele geprägt. Dazu gehören die Vivento der Deutschen Telekom (Reinle 2006; Skroblin 2005; vgl. auch Diedrich/ Bergström 2006), die DB JobService GmbH der Deutschen Bahn (Hüning/Stodt 1999; Messerschmidt 2001; Rehder 2003) sowie die Personalvermittlungsabteilung HVB Profil der damaligen HypoVereinsbank (Eurofound 2005). Als eigenständiges betriebliches Flexibilitätsinstrument wurden Abteilungen zur internen Personalvermittlung bisher kaum behandelt (Ausnahmen bilden Herrwig 2001; Klein-Schneider 2003; Kirsch/Mühge 2008, Mühge 2011; Wirth 2011). Vor diesem Hintergrund ist das Ergebnis der Recherchen überraschend, dass in wenigen Monaten insgesamt 51 Personalvermittlungsabteilungen in deutschen Unternehmen und Verwaltungen identifiziert werden konnten. Diese Anzahl ist ein erster Hinweis darauf, dass PVA in ihrer Bedeutung für die betriebliche Flexibilität tendenziell unterschätzt werden.

\section{Abb. 1: Personalvermittlungsabteilungen in öffentlicher und privater Wirtschaft - in \% -}

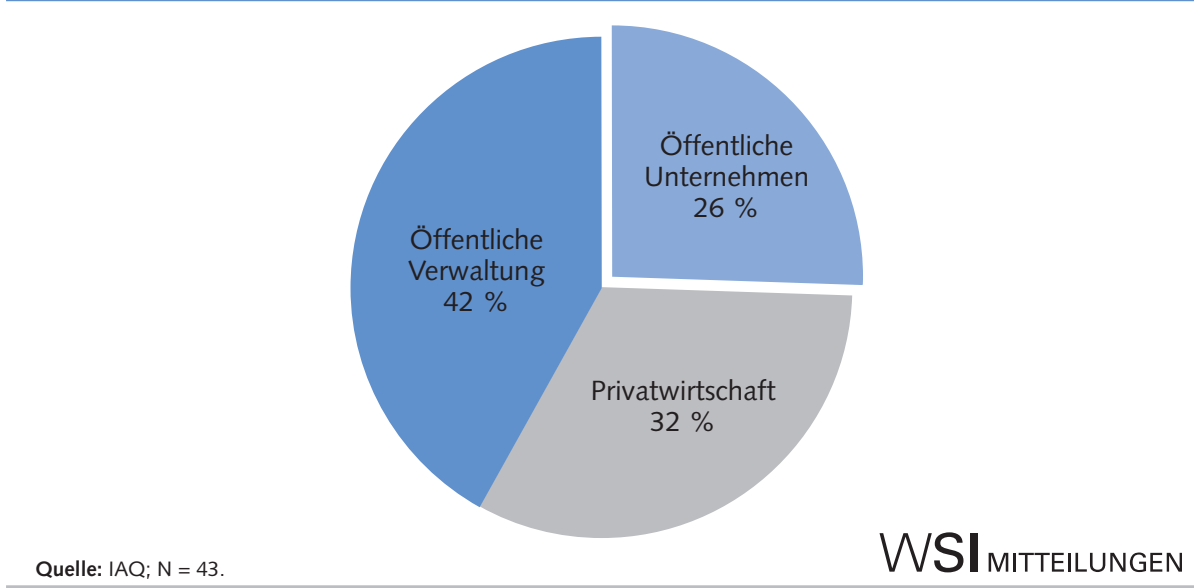

Ein zentraler Parameter, um die Bedeutung von Personalvermittlungsabteilungen insgesamt abzuschätzen, ist die Betriebsgröße. Die Befragungen zeigen, dass PVA vor allem von sehr großen Arbeitgebern und von Konzernbetrieben eingesetzt werden. Der Median der Befragung lag bei einer Betriebsgröße von 7.800 Beschäftigten, das arithmetische Mittel bei 39.000 Beschäftigten. Im genauen Wortlaut zielte unsere Frage darauf, wie viele Beschäftigte des Unternehmens im Fall von Arbeitsplatzverlust von den Leistungen der Abteilung zur internen Personalvermittlung profitieren. Damit wurden die Betriebsteile ausgeblendet, die Teil der Unternehmen unserer Stichprobe sind, für die aber die PVA und die Regeln zur Beschäftigungssicherung nicht gelten. Ein Beispiel: In der aktuellen Standortsicherung bei Siemens liegt die Sparte IT-Solutions/SIS nicht im Geltungsbereich der Siemens-Beschäftigungssicherung.

Aus dem so ermittelten Volumen an Beschäftigten konnten wir die quantitative Verbreitung der Personalvermittlungsabteilungen unserer Stichprobe ermitteln: Die Stichprobe erstreckt sich über insgesamt 1,25 Millionen Beschäftigte ( $\mathrm{N}=32$ Unternehmen/Verwaltungen). Das ist mehr als ein Fünftel (22\%) der gesamten Beschäftigung in Großbetrieben (mit mehr als 500 Beschäftigten) und bedeutet, dass jeder fünfte Beschäftigte in deutschen Großunternehmen in das beschäftigungssichernde Regelwerk der PVA aus unserer Stichprobe fällt und entsprechend arbeitsmarktpolitisch betreut wird, wenn der Verlust des Arbeitsplatzes erfolgt. ${ }^{3}$ Der Wert zeigt, dass heute Abteilungen zur internen Personal- vermittlung keine Exoten sind, sondern zum normalen Instrumentarium der internen Flexibilität in Großbetrieben zählen.

Welche weiteren strukturellen Charakteristika besitzen Personalvermittlungsabteilungen und die zugehörigen Unternehmen? Betrachtet man die sektorale Verteilung der uns bekannten PVA, fallen zunächst Schwerpunkte in verschiedenen Wirtschaftsbereichen auf. Dazu gehören die öffentliche Kernverwaltung (mit neun Personalvermittlungsabteilungen), Universitätskliniken/Universitäten (9) und Unternehmen der Energiewirtschaft (8); weitere 17 Personalvermittlungsabteilungen verteilen sich auf verschiedene Wirtschaftsbereiche in privaten Unternehmen (vgl. Mühge 2011). Aufgrund der unterschiedlichen Rahmenbedingungen ist es an dieser Stelle sinnvoll, interne Personalvermittlungen nach Unternehmenstypen zu unterscheiden: privatwirtschaftliche Unternehmen, Unternehmen privater Rechtsform im öffentlichen Eigentum (z.B. Stadtwerke oder die aus den ehemaligen Sondervermögen Bundesbahn und -post entstandenen Unternehmen) oder öffentliche Körperschaften bzw. Kernverwaltungen von Bund, Ländern und Kommunen. Abbildung 1 zeigt, das etwa zwei Drittel der PVA in der öffentlichen Wirtschaft und Verwaltung zu finden sind.

2 Es wurden 34 Interviews mit Leitungen und 30 Interviews mit Betriebs-/Personalräten realisiert. Ein Matching der Daten wurde nur in Bezug auf die Branchenzugehörigkeit vorgenommen $(\mathrm{N}=43)$.

3 Berechnung auf Basis der Befragungsdaten sowie der Beschäftigten- und Betriebsstatistik der Bundesagentur für Arbeit (Vergleichswerte zum Stand 31. Dezember 2006). 


\subsection{KÜNDIGUNGSSCHUTZ UND INTERNE PERSONALVERMITTLUNG}

Der hohe Anteil an öffentlichen Arbeitgebern legt die These nahe, dass Personalvermittlungsabteilungen im Zusammenhang mit dem im öffentlichen Sektor typischen hohen Kündigungsschutz stehen. ${ }^{4}$ Die folgenden Abschnitte behandeln die These, dass sie ein wichtiges Instrument sind, um bestehende Regeln zum betrieblichen Kündigungsschutz fortzuführen, die - etwa aufgrund von Strategien zur Personal(kosten)senkung - umstritten sind. Die Einführung der PVA ist Teil eines „Verhandlungspakets“ zwischen den Sozialpartnern, um Personalabbau zu ermöglichen, ohne das Niveau der Beschäftigungssicherheit abzusenken.

Ein erster Schritt zur Überprüfung der Hypothese war die Frage an die Interviewpartner, ob die Beschäftigten oder bestimmte Mitarbeitergruppen auf dem internen Arbeitsmarkt auf einem ,sehr hohen Niveau formal kündigungsgeschützt oder quasi unkündbar" sind. Dem stimmten 29 von 33 Befragten zu, d.h. in etwa $85 \%$ der Unternehmen und Verwaltungen unserer Stichprobe gibt es - zumindest für Teile der Beschäftigten - einen erhöhten Kündigungsschutz. Dieser erhöhte Kündigungsschutz kann sowohl auf Tarifverträgen, tariflichen Vereinbarungen oder Betriebs-/Dienstvereinbarungen basieren; im Bereich der öffentlichen Kernverwaltung können Gesetze oder rechtliche Rahmenbedingungen seine Basis bilden.

Im zweiten Schritt haben wir die Anzahl der Mitarbeiter ermittelt, die dieser spezifische Kündigungsschutz abdeckt, und deren Anteile an allen Beschäftigten berechnet. Diese Anteilswerte variieren von Fall zu Fall beträchtlich, sie bewegen sich über das gesamte mögliche Spektrum von 0 bis $100 \%$. Der Median des Anteils kündigungsgeschützter Mitarbeiter liegt bei $45 \%$, das arithmetische Mittel bei $47 \%$. Damit ist etwas weniger als die Hälfte der durch unsere Stichprobe erfassten Beschäftigten auf einem hohen Niveau vor Kündigung geschützt.

Die Frage, inwiefern die Personalvermittlungsabteilung Ergebnis von Verhandlungen um die Fortschreibung des bestehenden Kündigungsschutzes ist, wurde in einer gesonderten Befragung an Betriebsund Personalräte thematisiert. 25 der befragten Beschäftigtenvertreter $(\mathrm{N}=29)$ stimmten dieser These zu. Auf die Frage nach ihrem Verhandlungserfolg gaben 16 von 25 Personal- und Betriebsräten (64\%) an, dass sie ihr Verhandlungsziel „,voll und ganz" erreicht haben, in lediglich zwei Fällen wurden die Ziele „ganz und gar nicht“ erreicht. Die Angaben der Betriebsräte und des Managements von Personalvermittlungsabteilungen sind in dieser Frage konsistent: Die Vermeidung betriebsbedingter Kündigungen zählen 27 der befragten 34 Leitungen von Personalvermittlungsabteilungen zu ihren Kernaufgaben (,außerordentlich wichtiges Ziel“). Die quantitativen Daten zeigen damit, dass das Ziel, die Beschäftigungssicherung trotz eines beschleunigten internen Strukturwandels fortzuführen, eine zentrale Bedingung für die Gründung von PVA ist.

Die qualitativen Daten unserer Forschungsprojekte weisen in die gleiche Richtung: Wenn Unternehmen sich zu einer Personalabbaustrategie entscheiden, hat dies meist zur Konsequenz, dass die internen Regeln zur Beschäftigungssicherheit unternehmenspolitisch strittig werden. Die Gründung von Personalvermittlungsabteilungen ist Ergebnis der aus Reduktionsstrategien folgenden internen Verhandlungen; sie bewegen sich im Spannungsfeld von geplantem Personalabbau und sozialer Sicherheit. Damit sind PVA primär ein Instrument des Personalabbaus unter Bedingungen erschwerter externer Flexibilität. Als Verhandlungsergebnis sind Personalvermittlungsabteilungen von der Verhandlungsmacht der Personalund Betriebsräte bzw. dem Einfluss von Gewerkschaften auf die Arbeitsmarktbedingungen in Unternehmen und Verwaltungen abhängig: Dies erklärt auch ihre Existenz in Unternehmen ohne erhöhten Kündigungsschutz - hier entstehen sie durch die Einflussnahme der Mitbestimmung auf die Unternehmenspolitik oder als Resultat des Scheiterns konventioneller Personalabbaustrategien (vgl. Kirsch/ Mühge 2008).

\subsection{WIRKSAMKEIT}

Ein Aspekt, der sich direkt daran anschließt, ist die Zielerreichung von Personalvermittlungsabteilungen. Inwieweit ermöglichen sie eine Reduktion des Personals ohne Entlassungen auf den externen Arbeitsmarkt? Hierauf gibt der Anteil der internen Personalvermittlungen am Stellenabbau in den Unternehmen und Verwaltungen insgesamt eine Antwort. $\mathrm{Zu}$ diesem Zweck wurden in der Erhebung Stromgrößen von aus Sicht der Beschäftigten „unfreiwilligen“ Stellenverlusten abgefragt. Diese Stromgrößen bestehen aus Jahressummen von Beschäftigten, denen betriebsbedingt gekündigt worden ist, Beschäftigten, die über Aufhebungsverträge das Unternehmen verlassen haben, Auszubildenden, die nach Abschluss ihrer Ausbildung nicht übernommen wurden, sowie Mitarbeitern mit befristetem Arbeitsvertrag, die nach Vertragsende keine Verlängerung erhalten haben.

Um diese vier Größen in Vergleich zueinander setzen zu können, wurden spezifische Abgangsraten berechnet: Sie sagen aus, wie viele Abgänge oder Bewegungen pro Jahr auf 100 Beschäftigte im Bestand zu verzeichnen sind. Dazu wurden zunächst die Angaben aus den Datensätzen addiert, in denen sowohl Angaben zur Jahressumme der spezifischen Abgänge (Bezugsjahr 2006) als auch zur Anzahl der Beschäftigten vorlagen. Die aggregierten Jahressummen der Bewegungsdaten wurden anschließend ins Verhältnis zu den Bestandsdaten an Beschäftigten (Stichtag Ende 2006) gesetzt (vgl. Abbildung 2).

Es wird deutlich, dass das wichtigste Instrument bei Nutzung des externen Arbeitsmarkts zum Personalabbau der Aufhebungsvertrag ist: Statistisch gesehen, werden auf 100 Beschäftigte zum Stand Ende 2006 im Jahresverlauf 0,80 Aufhebungsverträge abgeschlossen. Hingegen sind betriebsbedingte Kündigungen in den Unternehmen der Stichprobe vollkommen unbedeutend; auf 100 Beschäftigte gab es lediglich 0,09 Entlassungen in dieser Form. Deutlich häufiger wird die Beendigung von befristeten Beschäftigungsverhältnissen praktiziert: Bezogen auf 100 Beschäftigte wurden in den Unternehmen im Sample im Jahr 2006 0,37 befristete Verträge nicht weiter verlängert; der Wert für Auszubildende, die nicht übernommen werden, beträgt 0,09 .

„Kündigungsschutz" ist hier ein Sammelbegrif für verschiedene Regelungen und Normen zum Schutz der Beschäftigten vor betriebsbedingten Kündigungen. Der Begriff sagt damit noch nichts über die Reichweite und die Qualität des Schutzes aus; unsere Fallstudien zeigen entsprechend zahlreiche Variationen in der Ausgestaltung. Unterschiede zeigen sich aber nicht nur von Fall zu Fall, auch innerhalb eines Unternehmens können verschieden Varianten des Kündigungsschutzes für unterschiedliche Beschäftigtengruppen koexistieren. 
Analog zum Verfahren bei den vier externen Abgangsarten sind die Zugänge an Beschäftigten in Personalvermittlungsabteilungen dargestellt. Auf 100 Beschäftigte Ende des Jahres 2006 kommen 1,56 Übergänge in die PVA - damit liegt dieser Wert um 0,28 Punkte über der Summe aller vier oben betrachteten Abgangsarten, die insgesamt 1,28 ergibt. $55 \%$ des „unfreiwilligen "Stellenverlusts in der Stichprobe wird demnach durch Personalvermittlungsabteilungen aufgefangen.

Abteilungen zur internen Personalvermittlung leisten einen wichtigen Beitrag zur Beschäftigungsstabilität und damit zur sozialen Sicherheit der Betroffenen. Aber sie sind damit nicht per se frei von Risiken. In Anlehnung an Flecker (2005, S. 86) haben wir in der Befragung der Personal- und Betriebsräte drei verschiedene Arten von Nachteilen unterschieden, die den Beschäftigten durch interne Arbeitsvermittlung drohen können. Dies sind Risiken durch Änderungen der Arbeitszeit, durch den Wechsel des Arbeitsorts sowie durch die Absenkung des Entgelts. Unsere Frage an die Beschäftigtenvertreter war, inwieweit die interne Personalvermittlung mit diesen Risiken einhergeht. Die Verteilung der Antworten zeigt Abbildung 3, in der wir den ,objektiven“ Risiken der internen Arbeitsvermittlung subjektive Risiken gegenübergestellt haben. Im Ergebnis gibt es deutliche Unterschiede zwischen den messbaren und den "gefühlten Härten“ von Arbeitsplatzverlust und interner Vermittlung. Die Beschäftigten erleben den Arbeitsplatzverlust als hart, unabhängig davon, ob sie in der Regel per Betriebs- oder Dienstvereinbarung vor Einkommenseinbußen oder vor Verschlechterungen hinsichtlich der räumlichen Situation, der Arbeitszeiten oder anderer Arbeitsbedingungen geschützt sind. Dieses Auseinanderklaffen von objektiv gegebenen und subjektiv wahrgenommenen Risiken zeigt, dass die Betreuungsarbeit der internen Vermittlungsorganisationen von hoher Sensibilität gekennzeichnet sein muss, da der Verlust des alten Arbeitsplatzes und der Übergang in das interne Vermittlungsverfahren von den betroffenen Beschäftigten - trotz der hohen Beschäftigungssicherheit - offensichtlich ähnlich einschneidend erlebt wird wie eine Entlassung auf den externen Arbeitsmarkt mit anschließend drohender Arbeitslosigkeit.

\section{Abb 2: Externe und interne Flexibilität - Anteil der Abgänge bzw. Versetzungen an den Beschäftigten 2006}

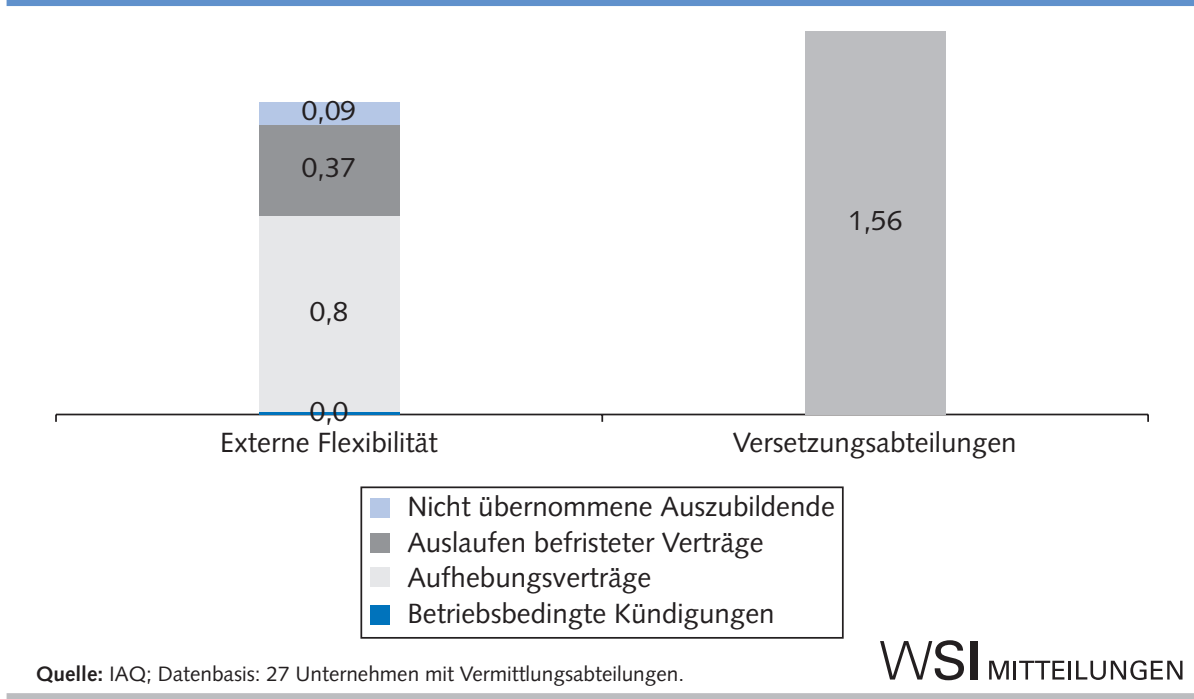

Die Mehrheit der Betriebs- und Personalräte kommt trotzdem zu dem Schluss, dass ihre PVA die Beschäftigten vor willkürlichen Personalentscheidungen bewahren und das negative Attribut „Abschiebebahnhof" nicht verdienen (Kirsch/Mühge 2010, zum Begriff „Abschiebebahnhof“ vgl. Köhler et al. 1989). Ein Grund dafür ist, dass die Abteilungen beim Personalabbau ein hohes Maß an prozessualer Sicherheit gewährleisten, weil Mindeststandards an Transparenz und Kommunikation für und mit den Beschäftigten eingeführt sind.

\section{Interessendivergenz bei der Personalauswahl}

Nimmt man unsere quantitativen Daten als Maßstab, sind die Ergebnisse und Leistungen von Personalvermittlungsabteilungen in der Summe als positiv zu bewerten. Die durch sie bereitgestellte Flexibilität hilft Unternehmen, Umbruchsituationen zu bewältigen, und den Beschäftigten, ein relativ hohes Maß an sozialer Sicherheit zu bewahren. Die quantitativen Daten sollen aber nicht darüber hinwegtäuschen, dass die Einführung und der Aufbau von PVA mit erheblichen innerorganisatorischen Konflikten verbunden sind. Wenn über Abteilungsgrenzen hinweg die interne Beschäftigtenmobilität gefördert werden soll, ziehen die Akteure, die in den Vermittlungsprozess involviert sind, nicht an einem Strang.

\subsection{DAS BASISDILEMMA DER INTERNEN ARBEITSVERMITTLUNG}

Wir vertreten die These, dass die Interessen der einzelnen Akteure in wesentlichen Punkten gegenläufig sind, obwohl die abteilungsübergreifende Vermittlung aus Sicht der Gesamtorganisation rational erscheint. Dieser Widerspruch manifestiert sich in den Kriterien der Personalauswahl. Die dabei beteiligten Akteure sind die Personalverantwortlichen der Abteilungen, die Personal abgeben, die Personalentscheider in aufnehmenden Abteilungen und das für die Vermittlung zuständige Personal der Personalvermittlungsabteilung.

Diese Konflikte und deren Folgen für den internen Arbeitsmarkt sollen im Folgenden auf Basis unserer qualitativen Arbeiten zu PVA beschrieben werden. Ausgangspunkt ist der Prozess des Stellenabbaus und der Stellenbesetzung. „Die Personalauswahl bei internen Personalbewegungen ist die Domäne der unteren Vorgesetzten" schreiben Köhler et al. (1989). Abteilungen zur internen Personalvermittlung sind aber - so unsere Hypothese - aufgrund ihrer Aufgabe gezwungen, die interne Personalauswahl zu ihrer Domäne zu machen. Auf diese Weise entsteht ein kompetitives Verhältnis zwischen verschiedenen Akteuren des internen Arbeitsmarkts.

Personalverantwortliche und Vorgesetzte vor Ort sind auf eine hohe Leistungsfähigkeit der ihnen untergebenen Mitarbeiter angewiesen, da ihre Arbeit 


\section{Abb. 3: Subjektive und objektive Risiken interner Personal- vermittlung für die Beschäftigten - in \% -}

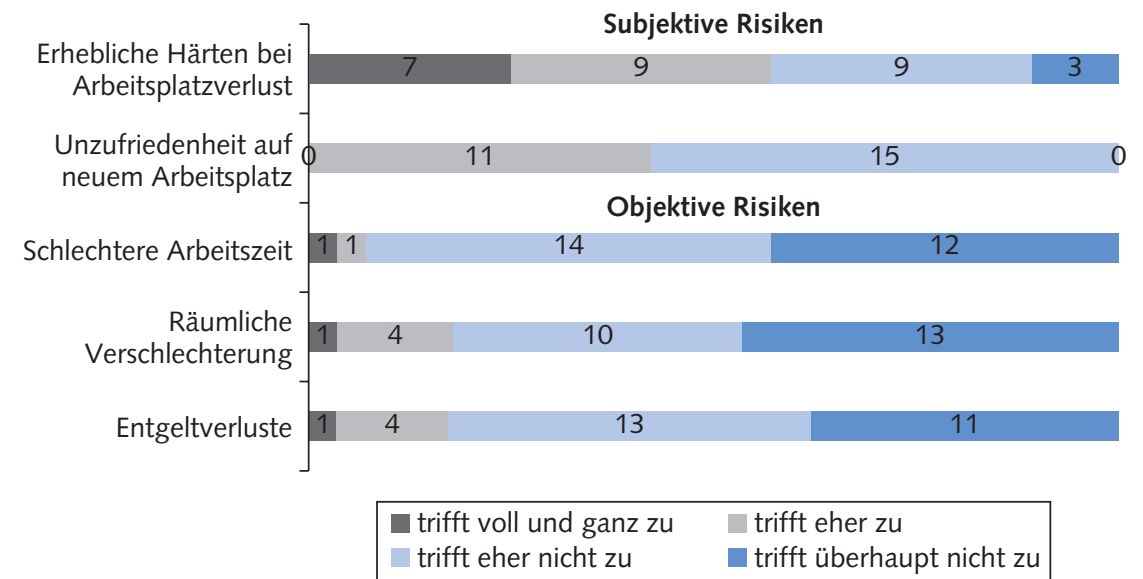

Quelle: IAQ; Befragung von Betriebs- und Personalräten.

WSI MITTEILUNGEN

und Ergebnisse zu großen Teilen nach Leistungskriterien beurteilt werden. Daher erfolgt auch die Personalauswahl nach Kriterien der Leistungsfähigkeit: Im Fall von Personalabbau sind die Personalverantwortlichen von dem Interesse geleitet, die Mitarbeiter mit hoher Leistungsfähigkeit $\mathrm{zu}$ halten und sich von den weniger leistungsstarken Beschäftigten zu trennen, sie also an die Personalvermittlungsabteilung abzugeben. Es erfolgt eine Negativauswahl nach Leistungsaspekten.

Die zweite Auswahlentscheidung findet in Abteilungen statt, die Bedarf an mehr Personal haben. Auch hier erfolgt die Auswahl der Mitarbeiter nach ihrer Leistungsfähigkeit, aber mit umgekehrten Vorzeichen: Leistungsstarke Beschäftigte sollen das zukünftige Ergebnis der Abteilung positiv beeinflussen. In Anlehnung an Windolf (1986, S. 237f.) werden zwei verschiedene Möglichkeiten unterschieden, Stellen zu besetzen. Die erste und übliche Möglichkeit beginnt mit der Suche nach Kandidaten, deren Profil am besten auf den Job passt: „The recruitment process usually begins with a definition of the ,ideal candidate " (ebd.). Die andere Möglichkeit ist die Besetzung der offenen Stelle mit abteilungseigenen Beschäftigten. Sie wird dann genutzt, wenn freie Stellen einen internen Karriereweg eröffnen sollen, etwa, um leistungsstarke Mitarbeiter zu motivieren oder an die Abteilung zu binden bzw. am Weggang zu hindern.

Richtet man die Perspektive auf die PVA, wird das Dilemma deutlich: Ihr Erfolg hängt $\mathrm{ab}$ von guten Kandidaten und von offenen Stellen, die sie mit ihren Beschäftigten bedienen kann. Inwiefern ihre Kandidaten vermittelbar sind und ob sie in Einstellungen zum Zuge kommen, ist direkt von den Auswahlkriterien und -entscheidungen abhängig. Wird die Auswahl allein den Personalverantwortlichen der Abteilungen überlassen, sind die offenen Stellen für die Kandidaten der Personalvermittlungsabteilung blockiert - sei es, weil die zu vermittelnden Beschäftigten aufgrund der ersten Personalauswahl in abgebenden Abteilungen nur schwer vermittelbar oder stigmatisiert sind (vgl. Wirth 2011), oder weil die offenen Stellen durch abteilungsinterne Aufstiege reserviert bleiben und damit die „Outsider“, hier die zu vermittelnden Kandidaten aus der PVA, diskriminiert werden (vgl. Windolf 1986, S. 238). „The process of selection is also a process of rejection. Decisions are made in favor of certain individuals but at the same time against others" (Kerr 1954).

Mit der Einführung von Personalvermittlungsabteilungen wird die Frage aufgeworfen, wer die Kontrolle über Personalentscheidungen hat und damit "seine“ Kandidaten - anstelle derer, die von der anderen Seite favorisiert werden - auf offene Stellen platzieren kann. Wir haben das Phänomen an anderer Stelle das „Basisdilemma der internen Arbeitsvermittlung“ genannt (Kirsch/Mühge 2008, 2010). Es drückt aus, dass die Personalauswahl bei Stellenabbau und -besetzung das entscheidende Handlungsfeld ist, um die Effektivität der PVA sicherzustellen.
Aus ihrer Sicht ist ein Regelwerk oder „Regime“ innerhalb des Unternehmens erforderlich, welches die Leistungsprinzipien bei der Personalauswahl abschwächt. Dies widerspricht den Interessen der Vorgesetzten vor Ort. Letztere wissen ferner, dass ihre Autonomie und Entscheidungsspielräume durch die Personalvermittlungsabteilungen verkleinert werden können, es in diesem Konflikt also auch um die Entscheidungskompetenz in Personalfragen geht. Die Kompetenz über Entlassung und Beförderung ist für Vorgesetzte ein entscheidendes Machtmittel (vgl. Ortmann et al. 1990, S. 18).

\subsection{KONSEQUENZEN FÜR PERSO- NALVERMITTLUNGSABTEILUNGEN}

Unsere qualitativen Daten zeigen, dass der faktische, aber auch der „gefühlte“ Verlust von Macht und Kompetenz erhebliche Kreativität freisetzt, um gefährdete Spielräume zu erhalten oder zurück zu erlangen. Die Abteilungsleitungen versuchen, über politisches Handeln innerhalb der Organisation ihre Interessen gegen die der Personalvermittlungsabteilung durchzusetzen. Um das Basisdilemma erfolgreich überwinden zu können und die abteilungsübergreifende Mobilität effektiv zu fördern, brauchen PVA also eine starke Position innerhalb des Unternehmens und auf dem internen Arbeitsmarkt. Dazu gehören hinreichende Macht-, Geld- und Personalressourcen. Die Bedeutung und Wirkung dieser Ressourcen lässt sich anhand von Beispielen illustrieren:

(1) Machtressourcen besitzen für Personalvermittlungsabteilungen eine zentrale Bedeutung. Wenn die Regeln der Personalallokation zugunsten der PVA geändert werden, braucht diese die Rückendeckung der Unternehmensspitze, um die neuen Regeln auch faktisch durchsetzen zu können. Eine weitere Ressource kann durch das „Verbot“ von Einstellungen aus dem externen Arbeitsmarkt entstehen, eine Regel, die die Vermittlungschancen der Bewerber aus der Personalvermittlungsabteilung deutlich erhöht, indem sie Konkurrenz „von außen“ ausschließt. Ferner sollte die Entscheidung über Ausnahmen vom Stopp externer Einstellungen in den Verantwortungsbereich der PVA fallen. Damit kontrolliert sie wichtige Ressourcen für die Abteilungen und bekommt eine gute Verhandlungsposition in Konflikten. 
Eine weitere Quelle für Ressourcen ist das interne Ausschreibungswesen von offenen Stellen: Es spricht viel dafür, die Meldung und Veröffentlichung offener Stellen in den Zuständigkeitsbereich der Personalvermittlungsabteilung zu verlagern, denn für eine effiziente Vermittlungsarbeit ist die unmittelbare Verfügung über alle stellenbezogenen Informationen wichtig.

(2) Zur Entschärfung des Basisdilemmas können der PVA zur Verfügung gestellte Geldressourcen einen wichtigen Beitrag leisten. Durch eine zeitlich befristete Subventionierung der Personalkosten können Anreize gesetzt werden, die die zu vermittelnden Beschäftigten attraktiv für die offenen Stellen anderer Abteilungen machen. Finanzielle Ressourcen benötigt die Personalvermittlungsabteilung auch dann, wenn ihre Klienten durch Qualifizierungsmaßnahmen fit für den internen Arbeitsmarkt werden sollen.

(3) Für eine professionelle Vermittlungsarbeit sind zudem hinreichende Personalressourcen erforderlich, um auf hohem fachlichen Niveau eine angemessene Beratung und andere arbeitsmarktbezogene Dienstleistungen für die betroffenen Beschäftigten zu gewährleisten.

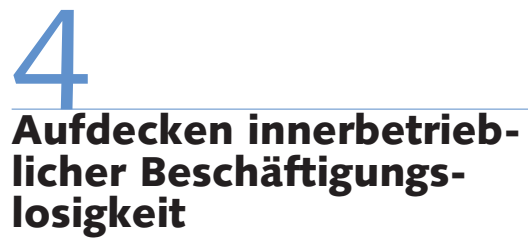

Die meisten PVA erheben grundsätzlich den Anspruch, ihre Beschäftigten vollständig auf interne Stellen zu vermitteln. Dieser Anspruch lässt sich in der Praxis jedoch nicht in vollem Umfang umsetzen, da ein Teil der Klientel keine Anschlussbeschäftigung auf dem internen Arbeitsmarkt finden kann. In dem Maße, in dem Anspruch und Wirklichkeit auseinanderklaffen, müssen Personalvermittlungsabteilungen sich mit dem Thema Arbeitslosigkeit im Unternehmen auseinandersetzen. Um dem rechtlichen Status der Teilnehmer von PVA gerecht zu werden - sie sind ja weiterhin per Arbeitsvertrag an das Unternehmen bzw. die Verwaltung gebunden und nicht im rechtlichen Sinne arbeitslos - verwenden wir im Folgenden (an Stelle von ,,interner Arbeitslosigkeit“) den Begriff ,interne Beschäftigungslosigkeit“.
Unsere Fallstudien zeigen einhellig, dass Personalvermittlungsabteilungen und Personalleitungen es vermeiden, die Begriffe Arbeitslosigkeit oder Beschäftigungslosigkeit im Zusammenhang mit dem internen Arbeitsmarkt zu benutzen, sei es, weil bei den Teilnehmern keine formale Arbeitslosigkeit vorliegt, oder weil der offene Umgang mit dem damit gemeinten Phänomen vermieden werden soll. Dessen ungeachtet haben unsere Forschungen deutlich gemacht, dass sich in PVA verschiedene Formen der Beschäftigungslosigkeit wiederfinden, die in ihren Strukturdimensionen der Arbeitslosigkeit auf dem externen Arbeitsmarkt entsprechen.

Zunächst kann man vereinfachend festhalten, dass die arbeitsmarktpolitischen Konzepte in Personalvermittlungsabteilungen im Kern auf die Behebung von sogenannter friktionaler oder Reibungsbeschäftigungslosigkeit zielen. Bei der Entwicklung der Vermittlungskonzepte ist man davon ausgegangen, dass grundsätzlich für alle Teilnehmer geeignete Zielarbeitsplätze vorhanden sind. Die Konzepte tragen diesem Anspruch Rechnung und zielen auf die Unterstützung der Teilnehmer in Übergängen, auf die Optimierung der Stellenbesetzungsprozesse und die Verkürzung der Vermittlungszeit. Die vermittlungsbezogenen Aktivitäten beziehen sich dementsprechend auf die Förderung der „mentalen Umstellung“ der betroffenen Mitarbeiter - für die auch kleinere Anpassungsqualifizierungen durchgeführt werden können - und auf die Optimierung von Such- und Auswahlprozessen.

Jedoch sind Personalvermittlungsabteilungen auch mit hartnäckigeren Formen der Beschäftigungslosigkeit konfrontiert: Dies gilt zum einen für strukturelle Beschäftigungslosigkeit (Begriffsbildung in Anlehnung an „strukturelle Arbeitslosigkeit" auf dem externen Arbeitsmarkt, vgl. Zerche et al. 2000, S. 119f.). Diese entsteht beispielweise dann, wenn bestimmte Berufsbilder im Unternehmen stark an Bedeutung verlieren oder ganz verschwinden. In der Mehrzahl der untersuchten Fälle sinkt die Bedeutung von Einfachtätigkeiten, zeitgleich steigt die Nachfrage nach höher qualifizierter Beschäftigung. Es handelt sich also nicht nur um reine Personalüberhänge, sondern um „Mismatch-Arbeitslosigkeit" auf internen Arbeitsmärkten. Offene Stellen können nicht besetzt werden, obwohl gleichzeitig Personalüberhänge bestehen.
Individuelle Merkmale, die einer Vermittlung im Wege stehen, sind ein weiterer Grund für eine hartnäckige Beschäftigungslosigkeit auf internen Arbeitsmärkten. Unsere quantitativen Daten werden durch Fallstudieninterviews bestätigt und ergeben, dass etwa $12 \%$ der Teilnehmer - und zwar unabhängig von ihrer Qualifikation - mit erheblichen individuellen Vermittlungshemmnissen behaftet sind. Genauso wie verfestigte Sockel- oder Langzeitarbeitslosigkeit zur Normalität des externen Arbeitsmarkts gehört, haben auch Personalvermittlungsabteilungen mit Teilnehmern zu tun, deren Vermittlung aus den genannten Gründen schwierig oder unrealistisch ist.

\section{Fazit}

Angesichts der geringen Aufmerksamkeit, die ihnen durch die (Fach-)Öffentlichkeit zukommt, ist es an der Zeit, Abteilungen zur internen Personalvermittlung als ein Regelinstrument großer Unternehmen und Verwaltungen wahrzunehmen. Wir haben auf Basis von quantitativen Befragungen belegt, dass die Organisation einer internen Arbeitsvermittlung ein hohes Maß an Beschäftigungssicherheit in Phasen von Restrukturierung und Personalabbau gewährleisten kann. Die Liste der positiven Eigenschaften und Ergebnisse von Personalvermittlungsabteilungen ließe sich weiter fortsetzen. Allerdings offenbart die Befragung auch die mit Arbeitsplatzverlust und anschließender Vermittlung verbundenen „Härten“. Diese zeigen sich weniger in den einzelnen greifbaren Risiken beruflicher Mobilität, wie z.B. Arbeitslosigkeit oder Entgeltverlust, sondern durch die vorgelagerten Auswahlprozesse und die immanenten Unsicherheiten des Stellenverlusts.

Weiterhin haben wir ausgeführt, dass sich Abteilungen zur internen Personalvermittlung dem Problem der internen Beschäftigungslosigkeit nicht verschließen können. Ein Teil ihrer Klienten ist schwer vermittelbar; für diese Gruppe gilt es, Lösungen zu entwickeln, die eine langfristige Verbesserung der Beschäftigungsfähigkeit anstreben, wenn die PVA zum „Sammelbecken" auch für dieses interne Beschäftigungssegment werden will.

Unsere qualitativen wie quantitativen Daten zeichnen in Bezug auf diese Probleme ein positives Bild, ohne einige grund- 
sätzliche Schwierigkeiten zu überdecken. In der Regel gelingt es den Akteuren der Personalvermittlungsabteilungen, geeignete Instrumente und Strukturen zu entwickeln, die einen internen "Abschiebebahnhof“ verhindern. Schwierigkeiten haben insbesondere junge Versetzungsabteilungen zu verzeichnen, die ihre internen Regeln der
Stellenbesetzung und des Stellenabbaus gegenüber den anderen Akteuren des internen Arbeitsmarkts erst einmal durchsetzen müssen. Wenn ihnen das gelingt, setzt ein Prozess ein, in dem die Personalvermittlungsabteilungen schrittweise ihren Einflussbereich und ihr Aufgabenspektrum ausweiten. Aktuelle Falluntersuchungen in reiferen und erfahrenen Personalvermittlungsabteilungen zeigen, dass diese mit der Zeit ein komplexes System von miteinander verzahnten, internen Serviceleistungen entwickeln und sie damit für viele Aufgaben der internen Personalpolitik und -entwicklung - über den Personalabbau hinaus - unersetzbar geworden sind.

\section{LITERATUR}

Bosch, G. (1990): Qualifizieren statt entlassen. Beschäftigungspläne in der Praxis, Opladen

Diedrich, A./Bergström, O. (2006): Developing Restructuring Practice - Workforce Reduction at a Large Swedish Telecommunications Company, Institute of Management of Innovation and Technology (IMIT) Report, Göteborg

European Foundation for the Improvement of Living and Working Conditions (Eurofound) (2005): TransFair and HVB Profil. The internal organisation of Hypovereinsbank's placement and temporary work agency, EMCC case study, Dublin

Flecker, J. (2005): Interne Flexibilisierung - Von der Humanisierungsvermutung zum Risikobefund, in: Kronauer, M./Linne, G. (Hrsg.):

Flexicurity. Die Suche nach Sicherheit in der Flexibilität, Berlin, S. 73-93

Herrwig, I. (2001): Personalentwicklung und interner Arbeitsmarkt, in: Bertelsmann-Stiftung/Faulth-Herkner \& Partner/Oechsler, W. A (Hrsg.): Leitfaden Systematisches Beschäftigungs-Management. Hilfestellung für ein modernes Personalmanagement, Gütersloh, S. 324-252 Hüning, H.;/Stodt, U. (1999): Regulierte Desintegration. Aspekte des internen Arbeitsmarkts bei der Deutschen Bahn AG, in: Nickel, H. M./ Völker, S./ Hüning, H. (Hrsg.): Transformation - Unternehmensorganisation - Geschlechterforschung, Opladen, S. 175-203

Kerr, C. (1954): The Balkanization of Labor Markets, in: Bakke, E. W. (Hrsg.): Labor Mobility and Economic Opportunity, Cambridge, S. $92-110$

Kirsch, J./Mühge, G. (2008): Unternehmensinterne Arbeitsvermittlung als Alternative zur Entlassung. Erste Fallstudien zur Wirksamkeit Interner Arbeitsmärkte, Blaue Reihe des Instituts Arbeit und Qualifikation 2008-1, Gelsenkirchen

Kirsch, J./Mühge, G. (2010): Die Organisation der Arbeitsvermittlung auf internen Arbeitsmärkten, Düsseldorf

Klein-Schneider, H. (Hrsg.) (2003): Interner Arbeitsmarkt. Beschäftigung und Personalentwicklung in Unternehmen und Verwaltungen, Frankfurt a. M.

Köhler, Ch./Nuber, Ch./Schultz-Wild, R. (1989): Rationalisierungsprozesse mit verdeckten Folgen, in: Köhler, Ch./ Preisendörfer, P. (Hrsg.): Betrieblicher Arbeitsmarkt im Umbruch. Analysen zur Mobilität, Segmentation und Dynamik in einem Großbetrieb, Frankfurt/New York, S. $209-231$
Messerschmidt, H. (2001): „....ich habe versucht, die Leute von Ost nach West zu bekommen...". Mobilitätsbereitschaft und Mobilitätsverhalten ostdeutscher MitarbeiterInnen in der DB AG, in: ZtG Bulletin 22, S. 39-56

Mühge, G. (2011): Organisationstheoretische Überlegungen zum Aufbau und zur Funktionsweise von Versetzungsabteilungen, in: VossDahm, D./Mühge, G./Schmierl, K./Struck, O. (Hrsg.): Qualifizierte Facharbeit im Spannungsfeld von Flexibilität und Stabilität, Wiesbaden, S. $99-122$

Ortmann, G./Windeler, A./Becker, A./ Schulz, H.-J. (1990): Computer und Macht in Organisationen. Mikropolitische Analysen, Opladen Rehder, B. (2003): Betriebliche Bündnisse für Arbeit in Deutschland. Mitbestimmung und Flächentarif im Wandel, Frankfurt a.M./New York Reinle, D. (2006): Im Schatten der Telekom, in: Die Mitbestimmung 12, S. $10-17$

Skroblin, J.-P. (2005): Arbeitnehmerorientiertes Coaching - Begleitung und Beratung beruflicher Entwicklungen im Kontext lebenslangen Lernens, in: Gillen, J./Dehnbostel, P./Elsholz, U. (Hrsg.): Kompetenzentwicklung in vernetzten Lernstrukturen - Konzepte arbeitnehmerorientierter Weiterbildung, Bielefeld, S. 89-101

Voss-Dahm, D./Mühge, G./Schmierl, K./Struck, O. (Hrsg.) (2011): Qualifizierte Facharbeit im Spannungsfeld von Flexibilität und Stabilität, Wiesbaden

Windolf, P. (1986): Recruitment, Selection, and Internal Labour Markets in Britain and Germany, in: Organization Studies 7 (3), S. 235-254 Wirth, C. (2011): Beschäftigungssicherung durch Arbeitsvermittlung im internen Arbeitsmarkt. Ergebnisse einer explorativen Studie: in: VossDahm, D./Mühge, G./Schmierl, K./Struck, O. (Hrsg.): Qualifizierte Facharbeit im Spannungsfeld von Flexibilität und Stabilität, Wiesbaden, S. $123-150$

Zerche, J./Schönig, W./Klingenberger, D. (2000): Arbeitsmarktpolitik und -theorie. Lehrbuch zu empirischen, institutionellen und theoretischen Grundfragen der Arbeitsökonomik, München 